OPEN ACCESS

Edited by:

Stefania Grimaudo

University of Palermo, Italy

Reviewed by:

Rosaria Maria Pipitone,

University of Palermo, Italy

Mustapha Najimi,

Catholic University of Louvain,

Belgium

*Correspondence.

Qing Ye

yeqing1998@gmail.com

Yutong Wang

yutongw@ccmu.edu.cn

tThese authors have contributed equally to this work

Specialty section:

This article was submitted to

Molecular Medicine,

a section of the journal

Frontiers in Cell and Developmental

Biology

Received: 29 October 2019

Accepted: 03 March 2020

Published: 24 March 2020

Citation:

Lv F, Li N, Kong M, Wu J, Fan Z,

Miao $D, X u Y$, Ye $Q$ and Wang $Y$

(2020) CDKN2a/p16 Antagonizes

Hepatic Stellate Cell Activation

and Liver Fibrosis by Modulating ROS

Levels. Front. Cell Dev. Biol. 8:176.

doi: 10.3389/fcell.2020.00176

\section{CDKN2a/p16 Antagonizes Hepatic Stellate Cell Activation and Liver Fibrosis by Modulating ROS Levels}

\author{
Fangqiao $\mathrm{Lv}^{1+}$, Nan $\mathrm{Li}^{2+}$, Ming Kong ${ }^{2 \dagger}$, Jun $\mathrm{Wu}^{3+}$, Zhiwen Fan ${ }^{4}$, Dengshun Miao \\ Yong $\mathrm{Xu}^{2,5}$, Qing Ye ${ }^{4 *}$ and Yutong Wang ${ }^{1 *}$
}

\begin{abstract}
' Department of Cell Biology, Municipal Laboratory for Liver Protection and Regulation of Regeneration, School of Basic Medical Sciences, Capital Medical University, Beijing, China, ${ }^{2}$ Key Laboratory of Targeted Intervention of Cardiovascular Disease and Collaborative Innovation Center for Cardiovascular Translational Medicine, Department of Pathophysiology, Nanjing Medical University, Nanjing, China, ${ }^{3}$ Department of Anatomy, Nanjing Medical University, Nanjing, China,

${ }^{4}$ Department of Pathology, The Affiliated Drum Tower Hospital, Nanjing University Medical School, Nanjing, China, ${ }^{5}$ Institute of Biomedical Research, Liaocheng University, Liaocheng, China
\end{abstract}

The lipid-storage hepatic stellate cells (HSC) play as pivotal role in liver fibrosis being able to trans-differentiate into myofibroblasts in response to various pro-fibrogenic stimuli. In the present study we investigated the role of CDKN2a/p16, a negative regulator of cell cycling, in HSC activation and the underlying mechanism. Levels of p16 were significantly down-regulated in activated HSCs isolated from mice induced to develop liver fibrosis compared to quiescent HSCs isolated from the control mice ex vivo. There was a similar decrease in p16 expression in cultured HSCs undergoing spontaneous activation or exposed to TGF- $\beta$ treatment in vitro. More important, p16 down-regulation was observed to correlate with cirrhosis in humans. In a classic model of carbon tetrachloride $\left(\mathrm{CCl}_{4}\right)$ induced liver fibrosis, fibrogenesis was far more extensive in mice with p16 deficiency (KO) than the wild type (WT) littermates. Depletion of p16 in cultured HSCs promoted the synthesis of extracellular matrix (ECM) proteins. Mechanistically, p16 deficiency accelerated reactive oxygen species (ROS) generation in HSCs likely through the p38 MAPK signaling. P38 inhibition or ROS cleansing attenuated ECM production in p16 deficient HSCs. Taken together, our data unveil a previously unappreciated role for p16 in the regulation of HSC activation. Screening for smallmolecule compounds that can boost p16 activity may yield novel therapeutic strategies against liver fibrosis.

Keywords: CDKN2a/p16, liver fibrosis, ROS, p38 MAPK, hepatic stellate cell

\section{INTRODUCTION}

Liver fibrosis is part of a host defense response taking place when the liver is exposed to a host of injuries such as corrosive chemicals, toxins, pathogens, ischemia, excessive nutrients, and certain medications (Friedman, 2010; Hernandez-Gea and Friedman, 2011; Luedde and Schwabe, 2011; Manmadhan and Ehmer, 2019; Spinnenhirn et al., 2019). Being a tightly regulated

Abbreviations: ECM, extracellular matrix; HSC, hepatic stellate cell; MAPK, mitogen activated protein kinase; ROS, reactive oxygen species; TGF, transforming growth factor. 
pathophysiological process, liver fibrosis contributes to the wound healing and repair of the injured liver parenchyma thus protecting the architectural and functional integrity of the liver. On the contrary, uncontrolled and aberrant liver fibrosis disrupts the normal hepatic structure and heralds such end-stage liver diseases as hepatocellular carcinoma (HCC) and cirrhosis (Seki and Schwabe, 2015). Decades of rigorous research have uncovered myofibroblast, a specialized cell type that possesses both a contractile phenotype (muscle-like) and the ability to produce and secrete extracellular matrix proteins (fibroblastlike), as the key mediator of liver fibrosis (Kisseleva, 2017). Although various types of cells, including the liver resident cells and circulating cells, have been proposed as the potential source of myofibroblasts during liver fibrosis, lineage tracing/fatemapping experiments performed in experimental animals have unequivocally demonstrated that hepatic stellate cells (HSCs), the lipid-storage cells tucked between the liver parenchyma and the sinusoidal endothelium, are the predominant origin from which myofibroblasts are derived from Mederacke et al. (2013).

A host of factors contribute to the trans-differentiation of HSCs to mature myofibroblasts, including transforming growth factor (TGF- $\beta$ ), platelet derived growth factor (PDGF-BB), high glucose, and reactive oxygen species (ROS) (Liu and Desai, 2015; Alzahrani et al., 2018; Hou and Syn, 2018; Jia et al., 2018). ROS production, similar to fibrogenesis, is also considered a host defense mechanism (Sies, 2015). Mounting evidence points to a link between excessive ROS accumulation and HSC activation. For instance, mice with deficiencies in one of the enzymes involved in the catalysis of ROS generation, including NADPH oxidase 1 (NOX1), NOX2, and NOX4, exhibit weaker liver fibrogenic response with attenuated HSC activation (Paik et al., 2011; Bettaieb et al., 2015; Lan et al., 2015). In contrast, deletion of Nrf2, the master regulator of anti-oxidative stress, leads to aggravated liver fibrosis in mice (Xu et al., 2008). In accordance, administration of antioxidants has been shown to alleviate liver fibrosis in experimental animals (Cho and Song, 2018; Li et al., 2018d; Chen et al., 2019). Among the myriad signaling pathways that regulate ROS production, the mitogen activated protein kinase (MAPK) pathway has been shown to play a key role. Hattori et al. have reported that a specific p38/MAPK inhibitor FR-167653 dampens HSC activation in cirrhotic rats (Hattori et al., 2007). Kluwe et al. (2010) have reported that mice deficient in JNK1/MAPK or treated with the pan-JNK inhibitor SP600125 are protected from liver fibrosis induced by $\mathrm{CCl}_{4}$ injection or bile duct ligation (BDL).

P16, encoded by CDKN2a, is a negative regulator of cell cycling (Rayess et al., 2012). We have previously shown that p16 knockout mice, when placed on a pro-steatotic diet, exhibit more severe lipid deposition, inflammatory infiltration, and ROS accumulation in the liver compared to the WT mice ( $\mathrm{Lv}$ et al., 2017). Here we report that p16 deficiency amplifies liver fibrosis in mice by enhancing HSC activation. Mechanistically, p16 deficiency activates p38/MAPK signaling to promote ROS production in HSCs. Therefore, out data provide a strong rationale for the screening of small-molecule compounds that can boost p16 activity in the therapeutic intervention of liver fibrosis.

\section{MATERIALS AND METHODS}

\section{Animals}

Male BABL/c mice with p16 KO ( $\left.16^{-/-}\right)$, ca. 6-8 weeks old, were used in these studies. To induce liver fibrosis, carbon tetrachloride $\left(\mathrm{CCl}_{4}, 1.0 \mathrm{~mL} / \mathrm{kg}\right.$ as $\left.50 \% \mathrm{vol} / \mathrm{vol}\right)$ or corn oil is administered to mice every other day for 7 days. Then the mice were sacrificed $24 \mathrm{~h}$ after the last injection and the liver samples were used for further analysis. Alternatively, liver fibrosis was induced by bile duct ligation (BDL) as previously described (Kong et al., 2019b). Briefly, the mice were anesthetized with inhalation of $4 \%$ isoflurane. The abdomen was open to expose the bile duct. A 5-0 suture was placed around the bile duct and two surgical knots were tied to ensure effective obstruction. The mice were sacrificed 2 weeks after the procedure. Plasma ALT/AST and liver hydroxyproline levels were determined with kits according to the manufacturer's protocol (Jiancheng Bioengineering, China). All animal experiments were approved by NJMU Intramural Ethics Committee on Animal Studies.

\section{Cell Culture}

Immortalized human HSC cells (LX-2, ATCC) were maintained in Dulbecco's modified Eagle's medium (DMEM; GibcoTM, Thermo Fisher Scientific, Waltham, MA, United States) supplemented with $10 \%$ fetal bovine serum (FBS; ExCell Bio, Genetimes, Shanghai, China) as previously described (Kong et al., 2019a). Cells were used typically between 3rd and 5 th passages. Cells were plated at the density of $3 \times 10^{5}$ cells/well in 6-well culture dishes. Primary stellate cells were isolated by collagenase $\mathrm{P}$ digestion and Density gradient centrifugation as previously described (Lu et al., 2019). Small interfering RNA (siRNA) targeting human Cdkn2a (5'-ACACCGCUUCUGCCUUUUCTT- $3^{\prime}$ ) and non-silencing RNA were transfected at final concentration of each $50 \mathrm{nM}$ into LX-2 cells using a Lipofectamine RNAi MAX kit (Thermo Fisher Scientific) according to the manufacturer's instructions. EdU analysis was performed using Cell-Light EdU Apollo643 in vitro Kit according the manufacturer's instruction (RiboBio, Guangzhou, China). CCK-8 analysis was performed using Cell Counting Kit- 8 according the manufacturer's instruction (Dojindo, Japan). TGF- $\beta$ was purchased from PeproTech. Prior to TGF- $\beta$ treatment, LX-2 cells were serum starved overnight. The next day, TGF- $\beta$ was diluted in serum-free media to treat the cells.

\section{Histochemical and Immunofluorescence Staining}

The specimens were collected after sacrificing the mice. Then they were fixed in $10 \%$ formalin and embedded in paraffin. Paraffin tissue sections were stained with Masson's trichrome or Sirius Red for histomorphometric assessment. The liver samples of cirrhotic patients in the form of tissue array (Alenabio, China) were collected from Tongxu County People's Hospital, China. The study was approved by the Ethics Committee of Tongxu County People's Hospital. The length of the biopsy 
A

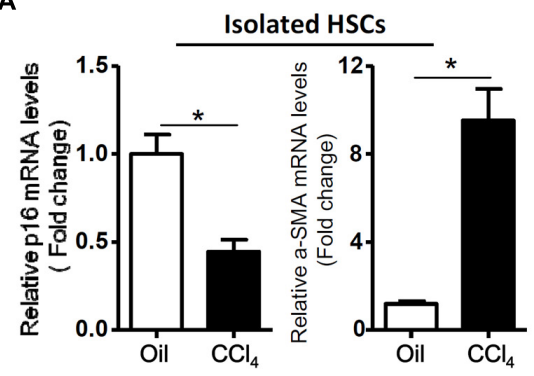

C

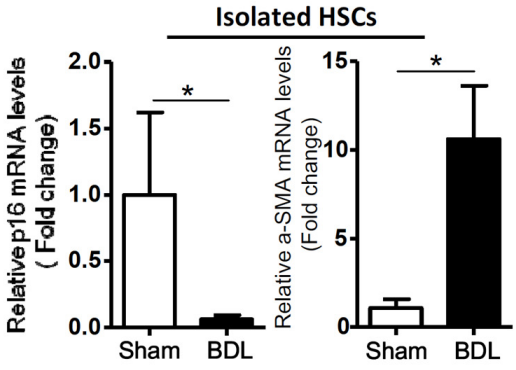

E

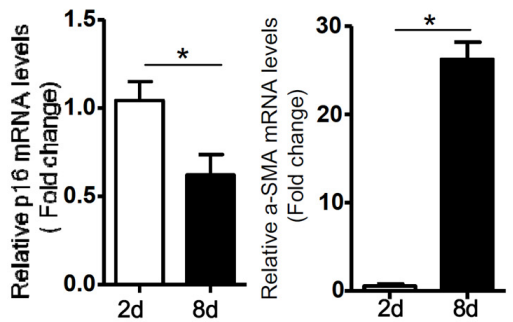

G

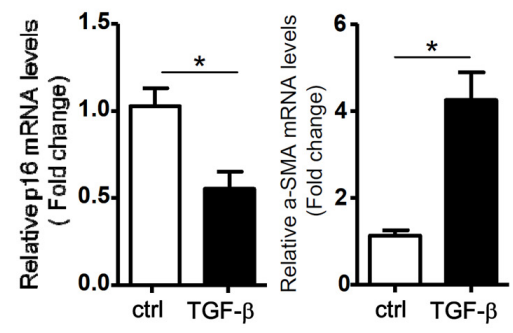

B

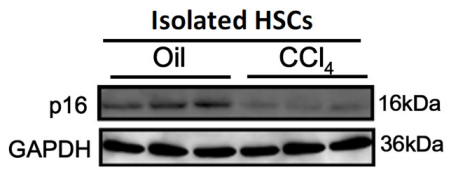

D

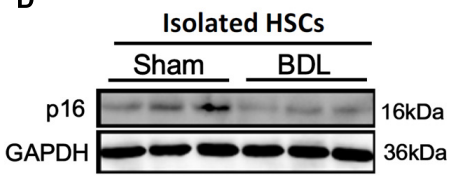

F

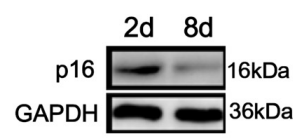

H

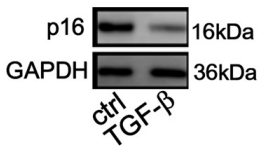

I

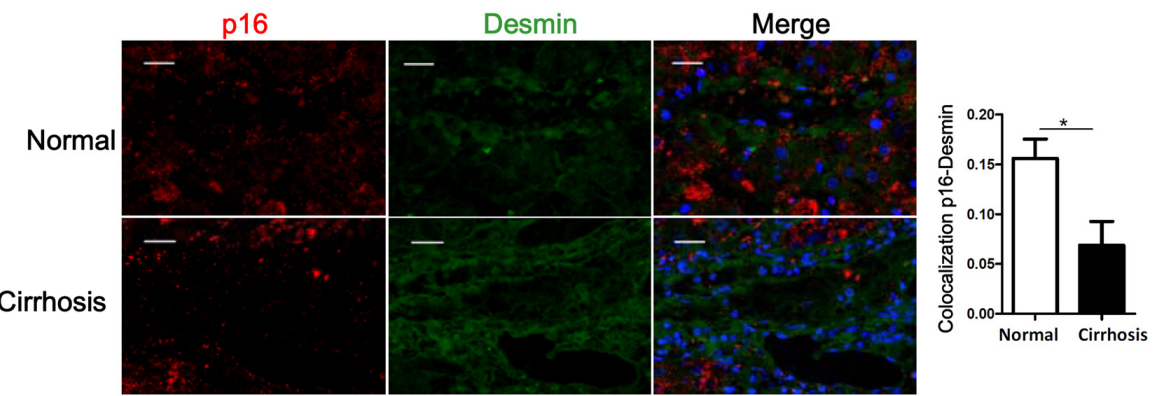

FIGURE 1 | p16 expression is down-regulated during HSC activation. (A,B) C57/BL6 mice were induced to develop liver fibrosis by $\mathrm{CCl}_{4}$ injection and primary HSCs were isolated as described in Methods. P16 expression was examined by qPCR and Western. (C,D) C57/BL6 mice were induced to develop liver fibrosis by BDL and primary HSCs were isolated as described in Methods. P16 expression was examined by qPCR and Western. (E,F) Primary mouse HSCs were isolated from C57/BL6 mice and allowed to undergo spontaneous activation. The cells were harvested at day 2 and day 8 and p16 expression was examined by qPCR and Western. (G,H) LX-2 cells were treated with or without TGF- $\beta(10 \mathrm{ng} / \mathrm{ml})$ for $48 \mathrm{~h}$. P16 expression was examined by qPCR and Western. Data represent averages of three independent experiments and error bars represent SEM. ${ }^{*} p<0.05$. (I) Normal and cirrhotic liver biopsy specimens were stained with anti-p16 and anti-desmin. $N=5$ for each group. Scale bar, $200 \mu \mathrm{m} .{ }^{*} p<0.05$. 
specimen is around $2 \mathrm{~mm}$. 5 cirrhosis samples and 5 healthy liver samples used as control were included in the present study. The patients' information is summarized in Supplementary Table S1. Sections were deparaffinized and dehydrated. For antigen retrieval, they were heating in a microwave in EDTA repairing buffer ( $\mathrm{pH}$ 9.0) for 15 min followed by blocking with $5 \%$ BSA. The sections were incubated with anti-p16 (1:200, Proteintech, catalog\# 10883-1) and anti-Desmin (1:200, Thermo Fisher Scientific, United States, catalog\# MA5-13259) overnight at $4^{\circ} \mathrm{C}$. After briefly washing, the sections were incubated with goat anti-rabbit IgG and goat anti-mouse IgG secondary antibodies and DAPI.

\section{Western Blot}

The protein was extracted from total cells or tissues using RIPA buffer containing PMSF and phosphatase inhibitor as previously described (Li et al., 2019a,b,c,d,e; Liu et al., 2019a,b; Lu et al., 2019; Shao et al., 2019; Weng et al., 2019; Yang et al., 2019a,b; Zhao et al., 2019). $30 \mu \mathrm{g}$ of protein were loaded in each lane and separated by $8 \%$ PAGE-SDS gel with allblue protein markers (Bio-Rad). Proteins were transferred to nitrocellulose membranes (Bio-Rad) in a Mini-Trans-Blot Cell (Bio-Rad). The membranes were blocked with 5\% fat-free milk powder in Tris-buffered saline at room temperature for half an hour and then incubated with the following primary antibodies at $4^{\circ} \mathrm{C}$ overnight: Western blot analyses were performed with anti-p16 (1:1,000, Proteintech, catalog\# 10883-1), antiCollagen type I (1:1,000, Proteintech, catalog\# 14695-1), anti- $\alpha-$ SMA (1:5,000, Abcam, catalog\# ab5694), anti-GAPDH (1:2,000, Proteintech, catalog\# 60004-1), anti-p38 (1:1,000, Proteintech, catalog\# 14064-1), and anti-phospho-p38 antibodies (1:2,000, Cell Signaling, catalog\# 1682). Image J software was used for densitometrical quantification and densities of target proteins were normalized to those of $\beta$-actin. Data are expressed as relative protein levels compared to the control group which is arbitrarily set as 1 .

\section{RNA Isolation and Real-Time RT-PCR}

Total RNA was extracted with Trizol reagent (Sigma, United States) from cells or tissues stored in a $-80^{\circ} \mathrm{C}$ freezer. Reverse transcriptase reactions were performed with $1 \mu \mathrm{g}$ of RNA using Reverse Transcription System (Promega, United States) as previously described (Fan et al., 2017, 2019; Li et al., 2017, 2018b,c,e,f,g; Yu et al., 2017; Liu et al., 2018; Yang et al., 2018; Zeng et al., 2018; Zhang et al., 2018; Kong et al., 2019a,b). Real-time RTPCR was performed with TransStart Green qPCR SuperMix (TransGen Biotech, Beijing, China). Primers were as follow: mouse p16: forward 5'-GGGTTTTCTTGGTGAAGTTCG$3^{\prime}$ and reverse $5^{\prime}$-TTGCCCATCATCATCACCT-3'; mouse $\mathrm{Col}$ I: forward 5' $5^{\prime}$ GACGCCATCAAGGTCTACTG-3' and reverse $5^{\prime}$ - ACGGGAATCCA-TCGGTCA-3'; mouse

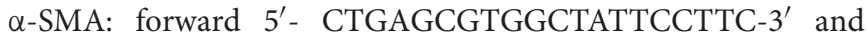
reverse 5'- CTTCTGCATCCTGTCAGCAA-3'; human p16: forward 5'-CGAATAGTTACGGTCGGAGG-3' and reverse 5'-TGAGAGTGGCGGGGTCG-3'; human Col I: forward $5^{\prime}$ - AGGCGAACAGGGCGACAGAG-3' and reverse 5'
GGCCAGGGAGACCGTTGAGT-3'; human $\alpha$-SMA: forward $5^{\prime}$ - CATCCTCCCTTGAGAAGAGTTA- $3^{\prime}$ and reverse $5^{\prime}$ TACATAGTGGTGCCCCCTGATA-3'; Ct values of target genes were normalized to the $\mathrm{Ct}$ values of housekeeping control gene (18s, 5'-CGCGGTTCTATTTTGTTGGT-3' and $5^{\prime}$-TCGTCTTCGAAACTCCGACT-3' for both human and mouse genes) using the $\Delta \Delta \mathrm{Ct}$ method and expressed as relative mRNA expression levels compared to the control group which is arbitrarily set as 1 .

\section{ROS Measurement}

LX-2 cells were cultured in 96-well plates and transfected with N.C. or siCDKN2A. $24 \mathrm{~h}$ after transfection, $2^{\prime}, 7^{\prime}$ dichlorodihydrofluorescein (DCF) was added to and incubated with the cells at $37^{\circ} \mathrm{C}$ for $30 \mathrm{~min}$. Frozen tissue sections were treated with autofluorescence quench for 5 min. After brief washing, the sections were incubated with the staining solution at $37^{\circ} \mathrm{C}$ for $30 \mathrm{~min}$. After 3 washes with $\mathrm{PBS}$, the cells or the sections were visualized with a fluorescence microscope (NIKON ECLIPSE C1).

\section{Statistical Analysis}

The data were calculated and expressed as mean SEM. Student $t$ tests were performed with GraphPad Prism. $p$ values smaller than 0.05 were considered significant.

\section{RESULTS}

\section{P16 Down-Regulation in HSCs Correlates With Liver Fibrogenesis}

We first tackled the question as to whether p16 levels would be altered during HSC activation. To this end, we used a series of different cell and animal models. In the first model wherein C57/BL6 mice were injected with $\mathrm{CCl}_{4}$ every other day for a week, p16 levels were significantly downregulated by $53 \%$ in the freshly isolated HSCs, accompanying an increase in $\alpha$-SMA levels, compared to those isolated from mice injected with corn oil (Figures 1A,B). In a second model in which the mice were subjected to bile duct ligation (BDL) to induce liver fibrosis, we again found that p16 levels were decreased by more than $85 \%$ in freshly isolated HSCs 2 weeks after the surgical procedure compared to the those isolated from the sham mice (Figures 1C,D). QPCR performed using whole liver homogenates revealed similar results showing a down-regulation of $\mathrm{p} 16$ and an up-regulation of $\alpha$-SMA (Supplementary Figure S1). Of interest, expression levels of check point kinase 1 (Chk1), a regulator of cell cycle, was not significantly altered in either isolated HSCs or in liver homogenates (Supplementary Figure S2).

We next isolated primary HSCs from normal C57/BL6 mice and allowed these cells to undergo spontaneous activation in vitro. As shown in Figures 1E,F, activated HSCs (8d) exhibited lower p16 expression levels than quiescent HSCs (2d). We also treated LX-2, an immortalized human HSC strain, with the profibrogenic growth factor TGF- $\beta$. Exposure of LX- 2 cells to TGF- $\beta$ 
similarly reduced p16 levels (Figures $\mathbf{1 G}, \mathbf{H}$ ). Of note, TGF$\beta$ treatment down-regulated p16 expression in LX-2 cells in a dose-dependent manner (Supplementary Figure S3).

Finally, we examined the levels of p16 in patients with cirrhosis. Compared to the healthy livers, p16 expression was significantly down-regulated in HSCs (identified by desmin) in cirrhotic livers (Figure 1). Collectively, these data suggest that there might be a correlation between p16 down-regulation and liver fibrogenesis both in vivo and in vitro.

\section{P16 Deficiency Promotes Liver Fibrogenesis in Mice}

Having observed that p16 down-regulation accompanies liver fibrogenesis, we hypothesized that p16 deletion in mice might accelerate the fibrogenic response. P16 deficient (KO) mice and their wild type (WT) littermates were injected with $\mathrm{CCl}_{4}$ to induced liver fibrosis. Masson's trichrome staining (Figure 2A) and picrosirius red staining (Figure $2 \mathbf{B}$ ) revealed that deposition

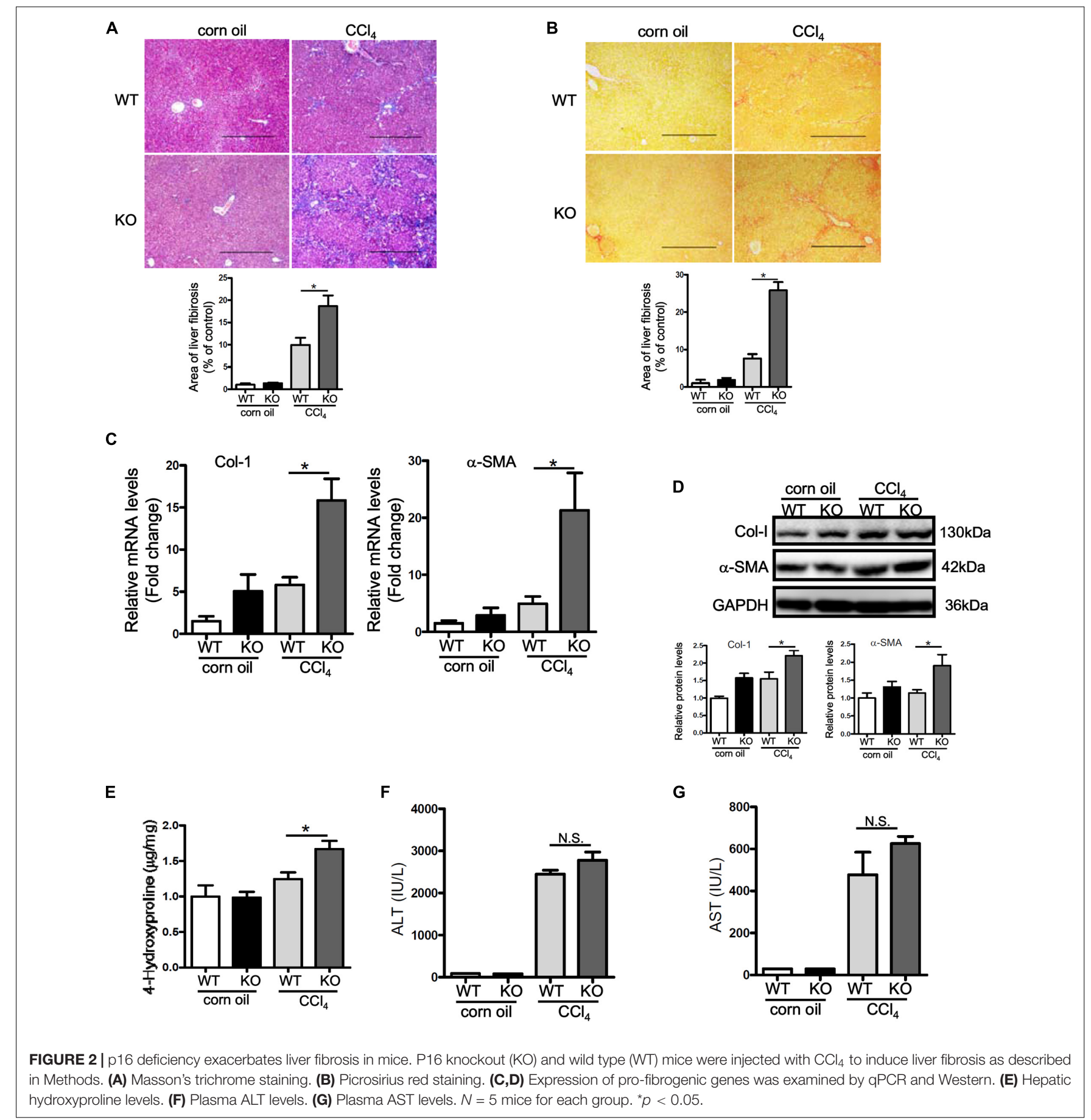


of ECM proteins was far more extensive in $\mathrm{KO}$ mice than in WT mice. Relative quantitative PCR (Figure 2C) and Western blotting (Figure 2D) confirmed that expression levels of profibrogenic genes, including collagen type I and $\alpha$-SMA, were higher in $\mathrm{KO}$ mice compared to WT mice. Relative quantitative PCR revealed that levels of interleukin 1 (Il-1) and interleukin 6 (Il-6) were higher in the KO livers than in the WT livers whereas levels of macrophage chemoattractant protein 1 (Mcp1) and tumor necrosis factor (Tnf) were comparable in the KO livers and in the WT livers (Supplementary Figure S4). Additional evidence to support p16 deficiency promoting liver fibrogenesis was provided by hepatic hydroxyl proline (HHP) quantification assay, which showed that increased HHP levels in KO mice than in WT mice (Figure 2E). Of interest, p16 deficiency appeared to have little impact on liver injury as evidenced by the observation that KO mice and WT mice exhibit comparable plasma ALT (Figure 2F) and AST (Figure 2G) levels, suggesting that the changes in liver fibrogenesis could be attributed to a HSCautonomous role for $\mathrm{p} 16$.

\section{P16 Deficiency Accelerates HSC Activation in vitro}

We next examined whether p16 deficiency in HSCs would be sufficient to stimulate its activation. Small interfering RNA (siRNA) mediated knockdown of p16 significantly up-regulated the expression of collagen type I and $\alpha$-SMA at both mRNA (Figure 3A) and protein (Figure 3B) levels in LX-2 cells.
A

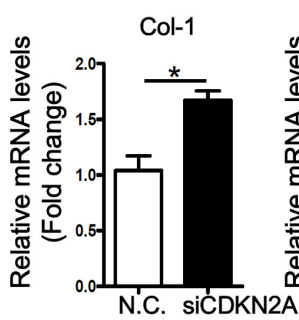

C
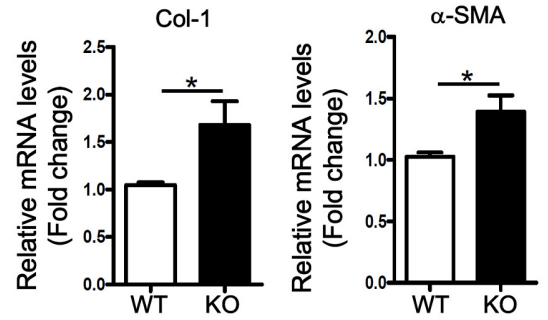

B

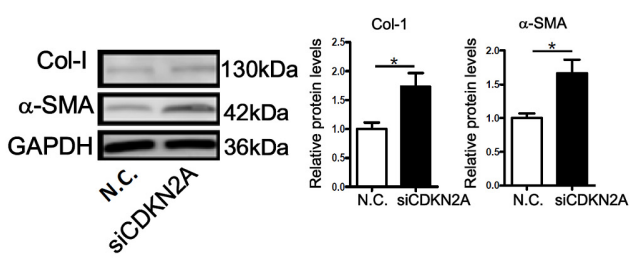

D

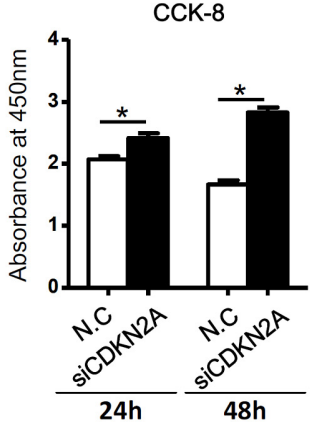

E

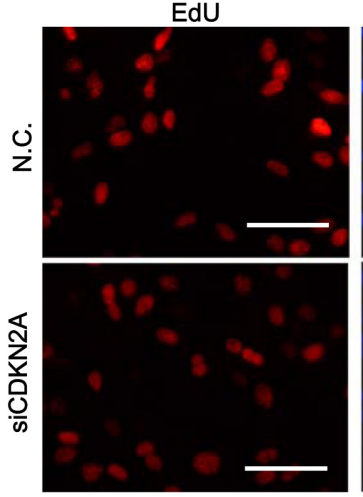

DAPI

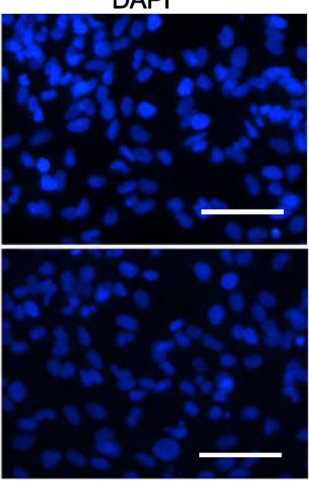

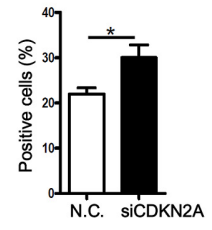

FIGURE 3 | p16 deficiency accelerates HSC activation. (A,B) LX-2 cells were transfected with siRNA targeting p16 or scrambled siRNA (SCR). Expression of pro-fibrogenic genes was examined by GPCR and Western. (C) Primary HSCs were isolated from p16 knockout (KO) and wild type (WT) mice and allowed to undergo spontaneous activation for 8 days. Expression of pro-fibrogenic genes was examined by qPCR. (D,E) LX-2 cells were transfected with siRNA targeting p16 $(50 \mathrm{nM})$ or scrambled siRNA (SCR). Cell proliferation was measured by CCK-8 activity and EdU incorporation as described in Methods. Scale bar, $50 \mu \mathrm{m}$. Data represent averages of three independent experiments and error bars represent SEM. ${ }^{*} p<0.05$. 
$\mathbf{A}$
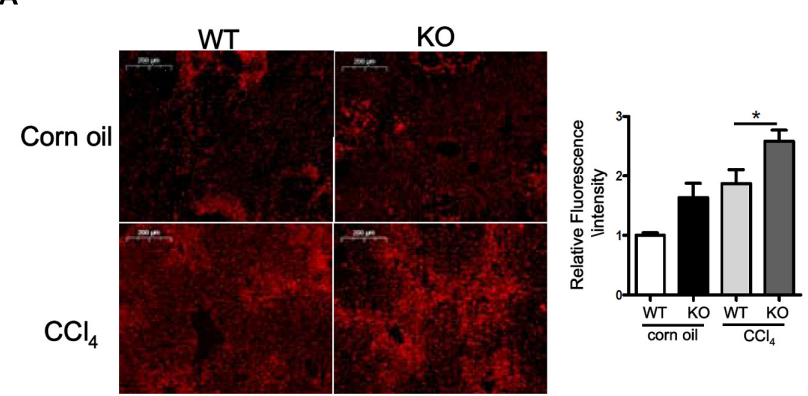

B

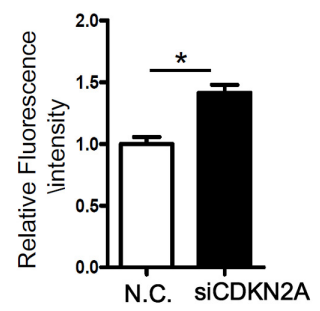

C
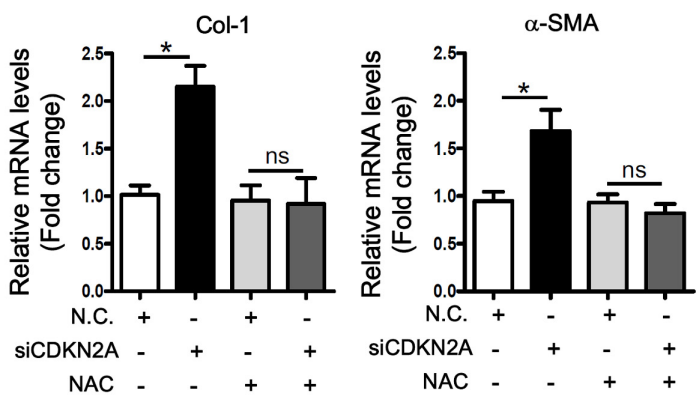

D
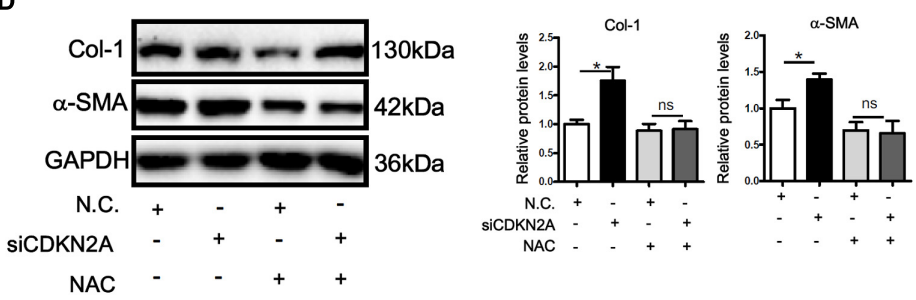

FIGURE 4 | p16 deficiency accelerates HSC activation by promoting ROS production. (A) P16 knockout (KO) and wild type (WT) mice were injected with CCl 4 to induce liver fibrosis as described in Methods. ROS levels were examined by DHE staining and quantified by Image Pro. Scale bar, $200 \mu \mathrm{m}$. (B) LX-2 cells were transfected with siRNA targeting p16 (50 nM) or scrambled siRNA (SCR). Measurement of ROS production was tested by DHE staining and quantified by Image Pro. $(\mathbf{C}, \mathbf{D})\llcorner X-2$ cells were transfected with siRNA targeting p16 or scrambled siRNA (SCR) followed by treatment with NAC. Expression of pro-fibrogenic genes was examined by $\mathrm{qPCR}$ and Western. Data represent averages of three independent experiments and error bars represent SEM. ${ }^{*} p<0.05$.

ELISA assay confirmed secreted collagen levels were also upregulated following p16 depletion (Supplementary Figure S5). In addition, activation of primary HSCs isolated from p16 KO mice was faster than those isolated from WT mice as evidenced by higher expression levels of collagen type I and $\alpha$-SMA (Figure 3C). HSC activation is characterized by, in addition to robust production of ECM proteins, enhanced proliferation. P16 knockdown significantly promoted proliferation of LX-2 cells as measured by CCK-8 levels (Figure 3D) and BrdU incorporation rate (Figure 3E).

\section{P16 Deficiency Promotes ROS Production During HSC Activation}

ROS accumulation plays a key role in HSC activation and liver fibrosis. We asked whether accelerated HSC activation as a result of p16 deficiency could be accounted for by ROS accumulation.
DHE staining showed that ROS levels were significantly higher in the livers of $\mathrm{CCl}_{4}$-injected mice than in the control livers; p16 deletion further up-regulated ROS levels in the fibrotic livers (Figure 4A). Similarly, p16 knockdown in LX-2 cells also increased ROS levels (Figure 4B). Next, we attempted to remove intracellular ROS by treating the cells with the antioxidant n-acetylcysteine (NAC). As shown Figures 4C,D, NAC treatment completely blunted the pro-fibrogenic effect of p16 depletion by normalizing the mRNA and protein levels of collagen type I and $\alpha$-SMA. Similar observation was made with regard to secreted collagen levels (Supplementary Figure S6).

\section{P38 MAPK Mediates ROS Production During HSC Activation}

MAPK signaling plays a critical role in ROS production (Son et al., 2011). Western blotting showed that activity of p38 MAPK, 


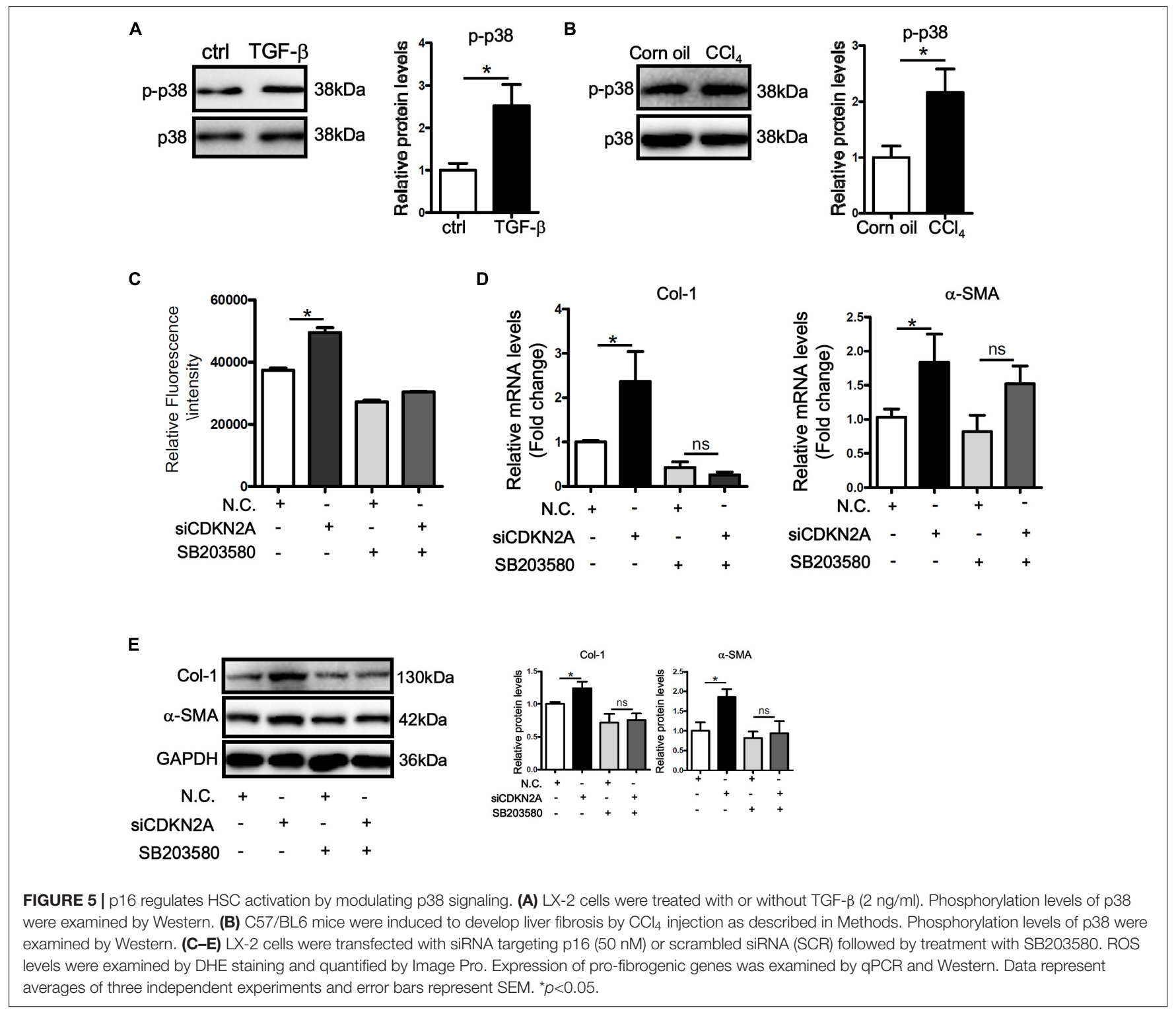

as judged by its phosphorylation status, was up-regulated by TGF- $\beta$ treatment in LX- 2 cells (Figure 5A). Similarly, p38 activity was higher in the fibrotic livers in $\mathrm{CCl}_{4}$-injected mice than in the control livers (Figure 5B). In accordance, treatment with a specific p38 inhibitor (SB203580) not only attenuated basal ROS levels in LX-2 cells but abrogated TGF- $\beta$ induced ROS production (Figure 5C). P38 inhibition also prevented the augmentation of collagen type I and $\alpha$-SMA following p16 knockdown at both mRNA (Figure 5D) and protein (Figure 5E) levels. Secreted collagen levels exhibited similar patterns as evaluated by ELISA (Supplementary Figure S7).

\section{DISCUSSION}

ROS-mediated activation of HSCs represents a paradigm in the pathogenesis of liver fibrosis. This notion is buttressed by a plethora of evidence (Paik et al., 2014; Crosas-Molist and Fabregat, 2015). Here we describe a pathway in which the cell cycling inhibitor CDKN2a/p16 regulates ROS production and HSC activation by modulating p38 activity. One of the better characterized functions of p16 is the regulation of cell cycling in which it acts to keep the cells in a quiescent state (Ruas and Peters, 1998). In the normal livers, HSCs are considered in a non-proliferative state having temporarily exited the cell cycle (Rippe and Brenner, 2004). Therefore, it makes sense that down-regulation of p16 expression during HSC activation may serve as the permissive step for the cells to exit the G0 phase. In addition to p16, there are other factors that can potentially antagonize liver fibrosis by forcing a quiescent state on the HSCs. For instance, statins exert their hepatoprotective effects by inducing the transcription factor KLF2, which in turn promotes NO production in liver sinusoidal endothelial cells. NO, via a paracrine mechanism, induces a 
quiescent phenotype in HSCs (Marrone et al., 2013). Sun et al. (2016) have reported that a SUMOylation dependent pathway represses SIRT1 transcription in quiescent HSCs, which allows the cells to resume proliferation thereby leading to liver fibrosis. Several independent reports have portrayed the nuclear receptor $\operatorname{PPAR} \gamma$ as a key regulator of HSC quiescence, either by inhibiting the pro-fibrotic JunD activity (Hazra et al., 2004), by inducing an adipogenic trans-differentiation program (She et al., 2005), or by suppressing ROS production (Hsu et al., 2013). Coincidently, both PPAR $\gamma$ and KLF2 have been shown, under various circumstances, to activate p16 expression (Guan et al., 1999; Wang et al., 2017). Because expression levels and/or activities of PPAR $\gamma$ and KLF2 are down-regulated during HSC activation (Marrone et al., 2015; Li et al., 2018a), it is reasonable to postulate that $\operatorname{PPAR} \gamma$ and KLF2 may contribute to suppression of HSC activation by restoring p16 expression. Our data thus suggest that p16 might be a key node in the intertwined network that regulates HSC phenotype and are consistent with the theme that maintenance of HSC quiescence is an attractive path through which antifibrotic strategies should be devised.

One intriguing question is whether ROS production is coupled to the regulation of cell cycling and, if so, whether p16 is moderator of these two inter-related processes. Indeed, mounting evidence suggests that cell cycling is programmed, at least in part, by the Redox status. It has been proposed that ROS-sensitive $\kappa$ of NF- $\kappa$ B probably provides the necessary cue for quiescent HSCs to re-enter the proliferative cycle (Sarsour et al., 2009). Of note, Ghiorzo et al. (2004) have shown that there is an inverse correlation between p16 expression and NF- $\kappa \mathrm{B}$ expression in melanoma tissues, suggesting that p16 down-regulation may be permissive in ROS accumulation and consequently HSC activation. This hypothesis can find support in our observation that inhibition of $\mathrm{p} 38$, which is upstream of and required for the non-canonical activation of NF-кB (Cristofanon et al., 2009), attenuated ROS accumulation and HSC activation. However, there is also evidence to suggest that NF- $\kappa$ B activates p16 expression (Chien et al., 2011). In addition, Jenkins et al. have reported that the ability of p16 to suppress ROS production is independent the cell cycling status (Jenkins et al., 2011). Therefore, more investigations should be conducted to reconcile these conflicting reports.

Hepatic stellate cell activation is not a strictly cell-autonomous process. In other words, non-HSCs including both intra- and extra-hepatic cells may influence the HSC phenotype. Since we have used a global CDKN2a knockout mouse strain in the present study, the possibility that p16 may exert cellspecific roles in regulating liver fibrosis cannot be ignored. For instance, p16 is known to suppress the production of proinflammatory cytokines in macrophages by promoting IRAK1 degradation (Murakami et al., 2012). Since pro-inflammatory mediators serve as a driving force for HSC activation, it is conceivable that the observed amelioration of liver fibrosis as a result of p16 deficiency could be attributed to a defect in macrophage-mediated inflammation. Similarly, hepatocyte death is considered a key factor promoting HSC activation (Brenner et al., 2013). p16 activation by $\beta$-catenin has been shown to protect hepatocytes from cell death, which may limit HSC activation (Fischer et al., 2007). Cell-specific p16 transgenic animal models may provide additional insights on the mechanism(s) by which p16 contributes to HSC activation and liver fibrosis.

\section{CONCLUSION}

In conclusion, our data point to a novel role for p16 as a suppressor of HSC trans-differentiation and liver fibrosis. The major limitations of the present study include the reliance on a single animal model, instead of multiple models, and an immortalized cell line (LX-2), as opposed to primary HSCs. Further studies are warranted to determine whether boosting p16 activity can be considered as a reasonable approach in the intervention of liver fibrosis.

\section{DATA AVAILABILITY STATEMENT}

The raw data supporting the conclusions of this article will be made available by the authors, without undue reservation, to any qualified researcher.

\section{ETHICS STATEMENT}

The studies involving human participants were reviewed and approved by the Capital Medical University Ethics Committee on Human Studies. The patients/participants provided their written informed consent to participate in this study. The animal study was reviewed and approved by Nanjing Medical University Ethics Committee on Humane Treatment of Experimental Animals.

\section{AUTHOR CONTRIBUTIONS}

YX and FL conceived the project and wrote the manuscript. FL, NL, MK, and YX designed the experiments. FL, NL, MK, JW, and ZF performed the experiments and collected the data. YW and QY secured funding. YW and QY provided supervision. DM provided key reagents.

\section{ACKNOWLEDGMENTS}

This work was supported in part by the 13th Five-year Plan of Nanjing Medical Science and Technology Development Fund (ZDX16006: Standardization Construction of Nanjing MultiCenter Biobank).

\section{SUPPLEMENTARY MATERIAL}

The Supplementary Material for this article can be found online at: https://www.frontiersin.org/articles/10.3389/fcell.2020.00176/ full\#supplementary-material 


\section{REFERENCES}

Alzahrani, B., Iseli, T., Ramezani-Moghadam, M., Ho, V., Wankell, M., Sun, E. J., et al. (2018). The role of AdipoR1 and AdipoR2 in liver fibrosis. Biochim. Biophys. Acta Mol. Basis Dis. 1864, 700-708. doi: 10.1016/j.bbadis.2017.12.012

Bettaieb, A., Jiang, J. X., Sasaki, Y., Chao, T. I., Kiss, Z., Chen, X., et al. (2015). Hepatocyte Nicotinamide Adenine Dinucleotide Phosphate Reduced Oxidase 4 Regulates Stress Signaling, Fibrosis, and Insulin Sensitivity During Development of Steatohepatitis in Mice. Gastroenterology 149, 468-480. e10. doi: 10.1053/j.gastro.2015.04.009

Brenner, C., Galluzzi, L., Kepp, O., and Kroemer, G. (2013). Decoding cell death signals in liver inflammation. J. Hepatol. 59, 583-594. doi: 10.1016/j.jhep.2013. 03.033

Chen, X., Xue, H., Fang, W., Chen, K., Chen, S., Yang, W., et al. (2019). Adropin protects against liver injury in nonalcoholic steatohepatitis via the Nrf2 mediated antioxidant capacity. Redox Biol. 21:101068. doi: 10.1016/j.redox. 2018.101068

Chien, Y., Scuoppo, C., Wang, X., Fang, X., Balgley, B., Bolden, J. E., et al. (2011). Control of the senescence-associated secretory phenotype by NFkappaB promotes senescence and enhances chemosensitivity. Genes Dev. 25, 2125-2136. doi: 10.1101/gad.17276711

Cho, Y. E., and Song, B. J. (2018). Pomegranate prevents binge alcohol-induced gut leakiness and hepatic inflammation by suppressing oxidative and nitrative stress. Redox Biol. 18, 266-278. doi: 10.1016/j.redox.2018.07.012

Cristofanon, S., Morceau, F., Scovassi, A. I., Dicato, M., Ghibelli, L., and Diederich, M. (2009). Oxidative, multistep activation of the noncanonical NF-kappaB pathway via disulfide Bcl-3/p50 complex. FASEB J. 23, 45-57. doi: 10.1096/fj. 07-104109

Crosas-Molist, E., and Fabregat, I. (2015). Role of NADPH oxidases in the redox biology of liver fibrosis. Redox Biol. 6, 106-111. doi: 10.1016/j.redox.2015.07. 005

Fan, Z., Li, N., Xu, Z., Wu, J., Fan, X., and Xu, Y. (2019). An interaction between MKL1, BRG1, and C/EBPbeta mediates palmitate induced CRP transcription in hepatocytes. Biochim. Biophys. Acta Gene Regul. Mech. 1862:194412. doi: 10.1016/j.bbagrm.2019.194412

Fan, Z., Li, Z., Yang, Y., Liu, S., Guo, J., and Xu, Y. (2017). HIF-1alpha coordinates epigenetic activation of SIAH1 in hepatocytes in response to nutritional stress. Biochim. Biophys. Acta Gene Regul. Mech. 1860, 1037-1046. doi: 10.1016/j. bbagrm.2017.08.002

Fischer, A. N., Fuchs, E., Mikula, M., Huber, H., Beug, H., and Mikulits, W. (2007). PDGF essentially links TGF-beta signaling to nuclear beta-catenin accumulation in hepatocellular carcinoma progression. Oncogene 26, 33953405. doi: $10.1038 /$ sj.onc. 1210121

Friedman, S. L. (2010). Evolving challenges in hepatic fibrosis. Nat. Rev. Gastroenterol. Hepatol. 7, 425-436. doi: 10.1038/nrgastro.2010.97

Ghiorzo, P., Mantelli, M., Gargiulo, S., Gramigni, C., Pastorino, L., Banelli, B., et al. (2004). Inverse correlation between p16INK4A expression and NF-kappaB activation in melanoma progression. Hum. Pathol. 35, 1029-1037. doi: 10.1016/ j.humpath.2004.02.017

Guan, Y. F., Zhang, Y. H., Breyer, R. M., Davis, L., and Breyer, M. D. (1999). Expression of peroxisome proliferator-activated receptor gamma (PPARgamma) in human transitional bladder cancer and its role in inducing cell death. Neoplasia 1, 330-339. doi: 10.1038/sj.neo.7900050

Hattori, S., Dhar, D. K., Hara, N., Tonomoto, Y., Onoda, T., Ono, T., et al. (2007). FR-167653, a selective p38 MAPK inhibitor, exerts salutary effect on liver cirrhosis through downregulation of Runx2. Lab Invest. 87, 591-601. doi: 10.1038/labinvest. 3700539

Hazra, S., Xiong, S., Wang, J., Rippe, R. A., Krishna, V., Chatterjee, K., et al. (2004). Peroxisome proliferator-activated receptor gamma induces a phenotypic switch from activated to quiescent hepatic stellate cells. J. Biol. Chem. 279, 1139211401. doi: $10.1074 / \mathrm{jbc} . \mathrm{m} 310284200$

Hernandez-Gea, V., and Friedman, S. L. (2011). Pathogenesis of liver fibrosis. Annu. Rev. Pathol. 6, 425-456. doi: 10.1146/annurev-pathol-011110-130246

Hou, W., and Syn, W. K. (2018). Role of Metabolism in Hepatic Stellate Cell Activation and Fibrogenesis. Front. Cell Dev. Biol. 6:150. doi: 10.3389/fcell.2018. 00150

Hsu, W. H., Lee, B. H., Hsu, Y. W., and Pan, T. M. (2013). Peroxisome proliferator-activated receptor-gamma activators monascin and rosiglitazone attenuate carboxymethyllysine-induced fibrosis in hepatic stellate cells through regulating the oxidative stress pathway but independent of the receptor for advanced glycation end products signaling. J. Agric. Food Chem. 61, 6873-6879. doi: $10.1021 / \mathrm{jf} 402082 \mathrm{~g}$

Jenkins, N. C., Liu, T., Cassidy, P., Leachman, S. A., Boucher, K. M., Goodson, A. G., et al. (2011). The p16(INK4A) tumor suppressor regulates cellular oxidative stress. Oncogene 30, 265-274. doi: 10.1038/onc.2010.419

Jia, Y., Wang, F., Guo, Q., Li, M., Wang, L., Zhang, Z., et al. (2018). Curcumol induces RIPK1/RIPK3 complex-dependent necroptosis via JNK1/2ROS signaling in hepatic stellate cells. Redox Biol. 19, 375-387. doi: 10.1016/j. redox.2018.09.007

Kisseleva, T. (2017). The origin of fibrogenic myofibroblasts in fibrotic liver. Hepatology 65, 1039-1043. doi: 10.1002/hep.28948

Kluwe, J., Pradere, J. P., Gwak, G. Y., Mencin, A., De Minicis, S., Osterreicher, C. H., et al. (2010). Modulation of hepatic fibrosis by c-Jun-N-terminal kinase inhibition. Gastroenterology 138, 347-359. doi: 10.1053/j.gastro.2009.09.015

Kong, M., Chen, X., Lv, F., Ren, H., Fan, Z., Qin, H., et al. (2019a). Serum response factor (SRF) promotes ROS generation and hepatic stellate cell activation by epigenetically stimulating NCF1/2 transcription. Redox Biol. 26:101302. doi: 10.1016/j.redox.2019.101302

Kong, M., Hong, W., Shao, Y., Lv, F., Fan, Z., Li, P., et al. (2019b). Ablation of serum response factor in hepatic stellate cells attenuates liver fibrosis. J. Mol. Med. 97, 1521-1533. doi: 10.1007/s00109-019-01831-8

Lan, T., Kisseleva, T., and Brenner, D. A. (2015). Deficiency of NOX1 or NOX4 prevents liver inflammation and fibrosis in mice through inhibition of hepatic stellate cell activation. PLoS One 10:e0129743. doi: 10.1371/journal. pone. 0129743

Li, M., Hong, W., Hao, C., Li, L., Wu, D., Shen, A., et al. (2018a). SIRT1 antagonizes liver fibrosis by blocking hepatic stellate cell activation in mice. FASEB J. 32, 500-511. doi: 10.1096/fj.201700612R

Li, N., Kong, M., Zeng, S., Xu, Z., Li, M., Hong, W., et al. (2018b). The chromatin remodeling protein BRG1 regulates APAP-induced liver injury by modulating CYP3A11 transcription in hepatocyte. Biochim. Biophys. Acta Mol. Basis Dis. 1864, 3487-3495. doi: 10.1016/j.bbadis.2018.08.003

Li, N., Li, M., Hong, W., Shao, J., Xu, H., Shimano, H., et al. (2018c). Brg1 regulates pro-lipogenic transcription by modulating SREBP activity in hepatocytes. Biochim. Biophys. Acta Mol. Basis Dis. 1864, 2881-2889. doi: 10.1016/j.bbadis. 2018.05.022

Li, R., Xin, T., Li, D., Wang, C., Zhu, H., and Zhou, H. (2018d). Therapeutic effect of Sirtuin 3 on ameliorating nonalcoholic fatty liver disease: the role of the ERK-CREB pathway and Bnip3-mediated mitophagy. Redox Biol. 18, 229-243. doi: 10.1016/j.redox.2018.07.011

Li, Z., Chen, B., Dong, W., Xu, W., Song, M., Fang, M., et al. (2018e). Epigenetic activation of PERP transcription by MKL1 contributes to ROS-induced apoptosis in skeletal muscle cells. Biochim. Biophys. Acta Gene Regul. Mech. 1861, 905-915. doi: 10.1016/j.bbagrm.2018.07.011

Li, Z., Chen, B., Weng, X., Yu, L., Song, M., Fang, M., et al. (2018f). The histone methyltransferase SETD1A regulates thrombomodulin transcription in vascular endothelial cells. Biochim. Biophys. Acta Gene Regul. Mech. 1861, 752-761. doi: 10.1016/j.bbagrm.2018.06.004

Li, Z., Zhang, X., Liu, S., Zeng, S., Yu, L., Yang, G., et al. (2018g). BRG1 regulates NOX gene transcription in endothelial cells and contributes to cardiac ischemia-reperfusion injury. Biochim. Biophys. Acta Mol. Basis Dis. 1864, $3477-$ 3486. doi: 10.1016/j.bbadis.2018.08.002

Li, M., Hong, W., Hao, C., Li, L., Xu, H., Li, P., et al. (2017). Hepatic stellate cellspecific deletion of SIRT1 exacerbates liver fibrosis in mice. Biochim. Biophys. Acta 1863, 3202-3211. doi: 10.1016/j.bbadis.2017.09.008

Li, Z., Chen, B., Dong, W., Kong, M., Fan, Z., Yu, L., et al. (2019a). MKL1 promotes endothelial-to-mesenchymal transition and liver fibrosis by activating TWIST1 transcription. Cell Death Dis. 10:899. doi: 10.1038/s41419-019-2101-4

Li, Z., Chen, B., Dong, W., Kong, M., Shao, Y., Fan, Z., et al. (2019b). The chromatin remodeler Brgl integrates ROS production and endothelial-mesenchymal transition to promote liver fibrosis in mice. Front. Dev. Cell Biol. 7:245. doi: 10.3389/fcell.2019.00245

Li, Z., Li, P., Lu, Y., Sun, D., Zhang, X., and Xu, Y. (2019c). A non-autonomous role of MKL1 in the activation of hepatic stellate cells. Biochim. Biophys. Acta Gene Regul. Mech. 1862, 609-618. doi: 10.1016/j.bbagrm.2019.03.001

Li, Z., Lv, F., Dai, C., Wang, Q., Jiang, C., Fang, M., et al. (2019d). Activation of galectin-3 (LGALS3) transcription by injurious stimuli in the liver is commonly mediated by BRG1. Front. Cell Dev. Biol. 7:310. doi: 10.3389/fcell.2019.00310

Li, Z., Xia, J., Fang, M., and Xu, Y. (2019e). Epigenetic regulation of lung cancer cell proliferation and migration by the chromatin 
remodeling protein BRG1. Oncogenesis 8:66. doi: 10.1038/s41389-0190174-7

Liu, L., Hong, W., Li, M., Ren, H., Wang, J., Xu, H., et al. (2019a). A Cross Talk Between BRG1 and Males Absent on the First Contributes to Reactive Oxygen Species Production in a Mouse Model of Nonalcoholic Steatohepatitis. Antioxid. Redox Signal. 30, 1539-1552. doi: 10.1089/ars.2016.6822

Liu, L., Mao, L., Wu, X., Wu, T., Liu, W., Yang, Y., et al. (2019b). BRG1 regulates endothelial-derived IL-33 to promote ischemia-reperfusion induced renal injury and fibrosis in mice. Biochim. Biophys. Acta Mol. Basis Dis. 1865, 2551-2561. doi: 10.1016/j.bbadis.2019.06.015

Liu, L., Wu, X., Xu, H., Yu, L., Zhang, X., Li, L., et al. (2018). Myocardinrelated transcription factor A (MRTF-A) contributes to acute kidney injury by regulating macrophage ROS production. Biochim. Biophys. Acta Mol. Basis Dis. 1864, 3109-3121. doi: 10.1016/j.bbadis.2018.05.026

Liu, R. M., and Desai, L. P. (2015). Reciprocal regulation of TGF-beta and reactive oxygen species: a perverse cycle for fibrosis. Redox Biol. 6, 565-577. doi: 10. 1016/j.redox.2015.09.009

Lu, Y., Lv, F., Kong, M., Chen, X., Duan, Y., Sun, D., et al. (2019). A cAbl-MRTF-A feedback loop contributes to hepatic stellate cell activation. Front. Cell Dev. Biol. 7:243. doi: $10.3389 /$ fcell.2019.00243

Luedde, T., and Schwabe, R. F. (2011). NF-kappaB in the liver-linking injury, fibrosis and hepatocellular carcinoma. Nat. Rev. Gastroenterol. Hepatol. 8, 108-118. doi: 10.1038/nrgastro.2010.213

Lv, F., Wu, J., Miao, D., An, W., and Wang, Y. (2017). p16 deficiency promotes nonalcoholic steatohepatitis via regulation of hepatic oxidative stress. Biochem. Biophys. Res. Commun. 486, 264-269. doi: 10.1016/j.bbrc.2017. 03.023

Manmadhan, S., and Ehmer, U. (2019). Hippo signaling in the liver - a long and ever-expanding story. Front. Cell Dev. Biol. 7:33. doi: 10.3389/fcell.2019. 00033

Marrone, G., Maeso-Diaz, R., Garcia-Cardena, G., Abraldes, J. G., Garcia-Pagan, J. C., Bosch, J., et al. (2015). KLF2 exerts antifibrotic and vasoprotective effects in cirrhotic rat livers: behind the molecular mechanisms of statins. Gut 64, 1434-1443. doi: 10.1136/gutjnl-2014-308338

Marrone, G., Russo, L., Rosado, E., Hide, D., Garcia-Cardena, G., Garcia-Pagan, J. C., et al. (2013). The transcription factor KLF2 mediates hepatic endothelial protection and paracrine endothelial-stellate cell deactivation induced by statins. J. Hepatol. 58, 98-103. doi: 10.1016/j.jhep.2012.08.026

Mederacke, I., Hsu, C. C., Troeger, J. S., Huebener, P., Mu, X., Dapito, D. H., et al. (2013). Fate tracing reveals hepatic stellate cells as dominant contributors to liver fibrosis independent of its aetiology. Nat. Commun. 4:2823. doi: 10.1038/ ncomms 3823

Murakami, Y., Mizoguchi, F., Saito, T., Miyasaka, N., and Kohsaka, H. (2012). p16(INK4a) exerts an anti-inflammatory effect through accelerated IRAK1 degradation in macrophages. J. Immunol. 189, 5066-5072. doi: 10.4049/ jimmunol.1103156

Paik, Y. H., Iwaisako, K., Seki, E., Inokuchi, S., Schnabl, B., Osterreicher, C. H., et al. (2011). The nicotinamide adenine dinucleotide phosphate oxidase (NOX) homologues NOX1 and NOX2/gp91(phox) mediate hepatic fibrosis in mice. Hepatology 53, 1730-1741. doi: 10.1002/hep.24281

Paik, Y. H., Kim, J., Aoyama, T., De Minicis, S., Bataller, R., and Brenner, D. A. (2014). Role of NADPH oxidases in liver fibrosis. Antioxid. Redox Signal. 20, 2854-2872. doi: 10.1089/ars.2013.5619

Rayess, H., Wang, M. B., and Srivatsan, E. S. (2012). Cellular senescence and tumor suppressor gene p16. Int. J. Cancer 130, 1715-1725. doi: 10.1002/ijc.27316

Rippe, R. A., and Brenner, D. A. (2004). From quiescence to activation: gene regulation in hepatic stellate cells. Gastroenterology 127, 1260-1262. doi: 10. 1053/j.gastro.2004.08.028

Ruas, M., and Peters, G. (1998). The p16INK4a/CDKN2A tumor suppressor and its relatives. Biochim. Biophys. Acta 1378, F115-F177.

Sarsour, E. H., Kumar, M. G., Chaudhuri, L., Kalen, A. L., and Goswami, P. C. (2009). Redox control of the cell cycle in health and disease. Antioxid. Redox Signal. 11, 2985-3011. doi: 10.1089/ARS.2009.2513

Seki, E., and Schwabe, R. F. (2015). Hepatic inflammation and fibrosis: functional links and key pathways. Hepatology 61, 1066-1079. doi: 10.1002/hep.27332

Shao, J., Weng, X., Zhuo, L., Yu, L., Li, Z., Shen, K., et al. (2019). Angiotensin II induced CSF1 transcription is mediated by a crosstalk between different epigenetic factors in vascular endothelial cells. Biochim. Biophys. Acta Gene Regul. Mech. 1862, 1-11. doi: 10.1016/j.bbagrm.2018.10.001
She, H., Xiong, S., Hazra, S., and Tsukamoto, H. (2005). Adipogenic transcriptional regulation of hepatic stellate cells. J. Biol. Chem. 280, 4959-4967. doi: 10.1074/ jbc.m410078200

Sies, H. (2015). Oxidative stress: a concept in redox biology and medicine. Redox Biol. 4, 180-183. doi: 10.1016/j.redox.2015.01.002

Son, Y., Cheong, Y. K., Kim, N. H., Chung, H. T., Kang, D. G., and Pae, H. O. (2011). Mitogen-activated protein kinases and reactive oxygen species: how can ROS activate MAPK pathways? J. Signal. Transduct. 2011:792639. doi: 10.1155/ 2011/792639

Spinnenhirn, V., Demgenski, J., and Brunner, T. (2019). Death Receptor Interactions With the Mitochondrial Cell Death Pathway During Immune Cell-, Drug- and Toxin-Induced Liver Damage. Front. Cell Dev. Biol. 7:72. doi: 10.3389/fcell.2019.00072

Sun, L., Fan, Z., Chen, J., Tian, W., Li, M., Xu, H., et al. (2016). Transcriptional repression of SIRT1 by protein inhibitor of activated STAT 4 (PIAS4) in hepatic stellate cells contributes to liver fibrosis. Sci. Rep. 6:28432. doi: 10.1038/ srep28432

Wang, C., Li, L., Duan, Q., Wang, Q., and Chen, J. (2017). Kruppellike factor 2 suppresses human gastric tumorigenesis through inhibiting PTEN/AKT signaling. Oncotarget 8, 100358-100370. doi: 10.18632/oncotarget. 22229

Weng, X., Zhang, Y., Li, Z., Yu, L., Xu, F., Fang, M., et al. (2019). Class II transactivator (CIITA) mediates IFN-gamma induced eNOS repression by enlisting SUV39H1. Biochim. Biophys. Acta Gene Regul. Mech. 1862, 163-172. doi: 10.1016/j.bbagrm.2019.01.005

Xu, W., Hellerbrand, C., Kohler, U. A., Bugnon, P., Kan, Y. W., Werner, S., et al. (2008). The Nrf2 transcription factor protects from toxin-induced liver injury and fibrosis. Lab. Invest. 88, 1068-1078. doi: 10.1038/labinvest. 2008.75

Yang, Y., Li, X., Peng, L., An, L., Sun, N., Hu, X., et al. (2018). Tanshindiol C inhibits oxidized low-density lipoprotein induced macrophage foam cell formation via a peroxiredoxin 1 dependent pathway. Biochim. Biophys. Acta Mol. Basis Dis. 1864, 882-890. doi: 10.1016/j.bbadis.2017.12.033

Yang, Y., Liu, L., Fang, M., Bai, H., and Xu, Y. (2019a). The chromatin remodeling protein BRM regulates the transcription of tight junction proteins: implication in breast cancer metastasis. Biochim. Biophys. Acta Gene Regul. Mech. 1862, 547-556. doi: 10.1016/j.bbagrm.2019.03.002

Yang, Y., Liu, L., Li, M., Cheng, X., Fang, M., Zeng, Q., et al. (2019b). The chromatin remodeling protein BRG1 links ELOVL3 trans-activation to prostate cancer metastasis. Biochim. Biophys. Acta Gene Regul. Mech. 1862, 834-845. doi: 10.1016/j.bbagrm.2019.05.005

Yu, L., Li, Z., Fang, M., and Xu, Y. (2017). Acetylation of MKL1 by PCAF regulates pro-inflammatory transcription. Biochim. Biophys. Acta Gene Regul. Mech. 1860, 839-847. doi: 10.1016/j.bbagrm.2017.05.006

Zeng, S., Wu, X., Chen, X., Xu, H., Zhang, T., and Xu, Y. (2018). Hypermethylated in cancer 1 (HIC1) mediates high glucose induced ROS accumulation in renal tubular epithelial cells by epigenetically repressing SIRT1 transcription. Biochim. Biophys. Acta Gene Regul. Mech. 1861, 917-927. doi: 10.1016/j.bbagrm. 2018.08.002

Zhang, X., Liu, S., Weng, X., Wu, T., Yu, L., Xu, Y., et al. (2018). Brg1 trans-activates endothelium-derived colony stimulating factor to promote calcium chloride induced abdominal aortic aneurysm in mice. J. Mol. Cell Cardiol. 125, 6-17. doi: 10.1016/j.yjmcc.2018.10.012

Zhao, Q., Yang, J., Chen, H., Li, J., Que, L., Zhu, G., et al. (2019). Peli1 induction impairs cardiac microvascular endothelium through Hsp90 dissociation from IRE1alpha. Biochim. Biophys. Acta Mol. Basis Dis. 1865, 2606-2617. doi: 10. 1016/j.bbadis.2019.06.017

Conflict of Interest: The authors declare that the research was conducted in the absence of any commercial or financial relationships that could be construed as a potential conflict of interest.

Copyright (c) $2020 \mathrm{Lv}, \mathrm{Li}$, Kong, Wu, Fan, Miao, Xu, Ye and Wang. This is an open-access article distributed under the terms of the Creative Commons Attribution License (CC BY). The use, distribution or reproduction in other forums is permitted, provided the original author(s) and the copyright owner(s) are credited and that the original publication in this journal is cited, in accordance with accepted academic practice. No use, distribution or reproduction is permitted which does not comply with these terms. 